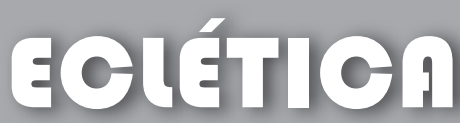 química
}

\section{SÍNTESE E CARACTERIZAÇÃO DE UM NOVO COMPLEXO BIMETÁLICO DE Pd(II) CONTENDO O ANTIBIÓTICO OXITETRACICLINA}

\author{
Wendell Guerra ${ }^{\prime *}$, Ana Paula Soares Fontes ${ }^{2}$, Elene Cristina Pereira-Maia ${ }^{3}$ \\ Instituto de Química, Universidade Federal de Uberlândia, 38.400-902 - Caixa Postal 593, Uberlândia - MG. \\ ${ }^{2}$ Departamento de Química, ICE, Universidade Federal de Juiz de Fora, 36036-330, Juiz de Fora - MG. \\ ${ }^{3}$ Departamento de Química - ICEx, Universidade Federal de Minas Gerais 31.270-901, Belo Horizonte - MG. \\ *e-mail:wg@iqufu.ufu.br
}

\begin{abstract}
Resumo: Este artigo descreve a síntese e caracterização de um novo complexo binuclear de paládio (II) contendo o antibiótico oxitetraciclina. O complexo foi caracterizado pelas técnicas usuais de análise. Pela análise do espectro de infravermelho sugerimos que o íon paládio(II) coordena-se no anel A, mais especificamente nos oxigênios da amida e da hidroxila vizinha no anel A e aos oxigênios da carbonila em C11 e da hidroxila em $\mathrm{C} 12$.
\end{abstract}

Palavras-chave: complexos de paládio, tetraciclinas, oxitetraciclina.

\section{Introdução}

Tetraciclinas constituem uma família de antibióticos de amplo espectro de atividade, que atuam como depressores da síntese proteíca. Suas excelentes propriedades antibacterianas aliada à baixa toxicidade, fizeram com que estes fármacos fossem durante muito tempo os antibióticos mais utilizados na clínica médica [1]. Contudo, o uso desses compostos no tratamento de infecções tem sido restringido devido ao aparecimento de uma resistência às tetraciclinas em um grupo variado de bactérias [2].

Recentemente nosso grupo de pesquisa publicou a síntese e caracterização de complexos de paládio com tetraciclinas que demonstraram atividade antibacteriana em bactérias resistentes à tetraciclina. Como exemplo, o complexo mononuclear de $\mathrm{Pd}(\mathrm{II})$ contendo tetraciclina, um complexo do tipo $\mathrm{PdLCl}_{2}$, foi dezesseis vezes mais ativo na linhagem E. coli HB101/PBR322 resistente a tetraciclina do que a droga livre [3]. Também demonstramos que complexos de $\mathrm{Pd}(\mathrm{II})$ contendo tetraciclinas apresentam atividade antitumoral [4].

No que se refere aos complexos de paládio, estes têm sido investigados quanto ao seu potencial biotecnológico, principalmente como novos agentes antibacterianos e antitumorais [5].

Em relação à química de coordenação das tetraciclinas, é sabido que tetraciclinas coordenam - se a íons metálicos presentes nos meios biológicos, sendo que sua biodisponibilidade é afetada pela coordenação [1-3]. De certa forma, isto tem motivado estudos envolvendo a química de coordenação de íons metálicos às tetraciclinas em vários grupos de pesquisa, mas devido à complexidade da estrutura molecular das tetraciclinas e ao grande número de sítios potencialmente complexantes, tem havido muita controvérsia na localização destes sítios [6-19].

Continuando nossos trabalhos, reportamos neste artigo parte da estratégia do nosso grupo de pesquisa, que é a obtenção e caracterização de possíveis novos agentes terapêuticos pela coordenação de metais de transição as tetraciclinas. Para 
efeitos de comparação, preparamos também um novo complexo mononuclear de $\mathrm{Pd}(\mathrm{II})$ contendo oxitetraciclina como ligante utilizando um método de síntese já descrito por nós [3].

\section{Material e métodos}

Todos reagentes foram usados sem purificação prévia.

Os espectros na região do IV foram feitos num espectrofotômetro Perkin-Elmer 283 B, na região de 4000 a $200 \mathrm{~cm}^{-1}$ utilizando como suporte pastilhas de CsI.

Os espectros de absorção na região do UV-Vis foram feitos num espectrofotômetro duplo feixe CARY 100 VARIAN.

Os espectros de $\mathrm{RMN}$ de ${ }^{1} \mathrm{H}$ foram feitos no LAREMAR/UFMG utilizando um espectrômetro Brucker Avance DRX 400. Os deslocamentos químicos foram expressos em $\delta$ a partir do padrão interno TMS.

Os resultados de análise elementar foram obtidos num Perkin-Elmer $2400 \mathrm{CHN}$. As análises por absorção atômica foram feitas num espectrofotômetro Hitachi 8200.

As análises de condutividade foram feitas num aparelho Digimed DM 31 usando uma célula de constante $1,06 \mathrm{~cm}^{-1}$, dimetilformamida como solvente $\left(\Lambda_{\mathrm{M}}=1.22 \mu \mathrm{s} / \mathrm{cm}^{-1}\right)$ e brometo de tetraetilamônio como um padrão $\left(\Lambda_{\mathrm{M}}=79,56 \mu \mathrm{s} / \mathrm{cm}^{-1}\right)$.

As análises termogravimétricas (TG/DTG) foram feitas num TG-50 METLLE STARE usando 6 $\mathrm{mg}$ de amostra. A amostra foi aquecida a $10^{\circ} \mathrm{C} / \mathrm{min}$ da temperatura ambiente até $850^{\circ} \mathrm{C}$, em atmosfera de oxigênio (taxa de fluxo $=200 \mathrm{~mL} / \mathrm{min}$ ). Os difratogramas de raios-X de pó foram registrados num aparelho Rigaku Geiger-flex 2037, em ângulos de $2 \theta$ variando de 0 a $90^{\circ}$.

\section{Síntese dos complexos}

\section{Complexo I (mononuclear)}

A uma solução de $0,5 \mathrm{mmol}$ do ligante em 5 $\mathrm{mL}$ de água deionizada, foi adicionado $0,5 \mathrm{mmol}$ de $\mathrm{K}_{2} \mathrm{PdCl}_{4}$ também dissolvido em $5 \mathrm{~mL}$ água deionizada. A mistura reacional foi mantida sob agitação durante 30 minutos, sob proteção da luz. O precipitado amrelo foi filtrado e lavado com água deionizada e etanol sendo posteriormente colocado num dessecador sob vácuo para secagem.
IR $v_{\max } \mathrm{KBr}\left(\mathrm{cm}^{-1}\right): 3413(\mathrm{OH}), 2880(\mathrm{CH})$, $1630(\mathrm{C}=\mathrm{O}), 1616(\mathrm{C}=\mathrm{O}), 1585(\mathrm{C}=\mathrm{O}), 1456,1397$, 1326, 1238, 1212, 1178, 1129, 1038, 1009, 1092, 943, 838, 772, 709, 725, 679, 641, 595, 511, 328 (Pd$\mathrm{Cl}), 302$ (Pd-Cl). Rendimento: 87\%. \% calculada para $\left[\mathrm{Pd}\left(\mathrm{C}_{22} \mathrm{H}_{24} \mathrm{~N}_{2} \mathrm{O}_{9} \mathrm{Cl}_{2}\right)\right] \cdot 2 \mathrm{H}_{2} \mathrm{O}$ : carbono $(39,20)$, hidrogênio $(4,19)$, nitrogênio $(4,16)$, paládio $(14,97)$. $\%$ encontrada: carbono $(39,25)$, hidrogênio $(4,23)$, nitrogênio $(4,11)$, paládio $(14,91)$. Cor: amarelo

\section{Complexo II (binuclear)}

$0,326 \mathrm{~g}$ de $\mathrm{K}_{2} \mathrm{PdCl}_{4}(1 \mathrm{mmol})$ foi dissolvido em $10 \mathrm{~mL}$ de água e adicionado a uma solução aquosa de oxitetraciclina $(0,5 \mathrm{mmol}) \mathrm{em} \mathrm{pH}$ fixo igual a 3,5 . Decorridos 40 minutos do início da reação o $\mathrm{pH}$ foi elevado a 6,5 pela adição de $\mathrm{NaOH}(\mathrm{aq})$ e a reação prosseguiu por 6 horas em $\mathrm{pH}$ controlado. $\mathrm{O}$ sólido formado foi filtrado e lavado com etanol e água e secado sob vácuo.

IR $v_{\max } \mathrm{KBr}\left(\mathrm{cm}^{-1}\right): 3454(\mathrm{OH}), 2928(\mathrm{CH})$, $1637(\mathrm{C}=\mathrm{O}), 1616(\mathrm{C}=\mathrm{O}), 1448,1372,1327,1257,1132$, 1066, 1019, 950, 895, 862, 834, 780, 724, 620, 583, 487, 330 (Pd-Cl). Rendimento: 82\%. \% Calculada para $\left[\mathrm{Pd}_{2}\left(\mathrm{C}_{22} \mathrm{H}_{26} \mathrm{O}_{11} \mathrm{~N}_{2}\right) \mathrm{Cl}_{2}\right] \cdot \mathrm{H}_{2} \mathrm{O}$ : carbono (32,38), hidrogênio $(3,43)$, nitrogênio $(3,43)$ paládio $(26,12) . \%$ encontrada: carbono $(32,15)$, hidrogênio $(3,39)$ nitrogênio $(3,38)$, paládio $(25,99)$. Cor: castanho

\section{Resultados e Discussão}

O ligante utilizado neste trabalho, oxitetraciclina, foi adquirido comercialmente e como caracterização foram realizados seus espectros de infravermelho e UV-Vis, assim como estudos de análise termogravimétrica (TG/DTG e DSC) para posterior comparação com os complexos obtidos.

A oxitetraciclina possui uma estrutura química muito interessante, podendo formar quelatos em várias posições devido à presença de vários grupos doadores $(\mathrm{N}, \mathrm{O})$. Os sítios de coordenação incluem os oxigênios em $\mathrm{C} 10-\mathrm{C} 12$, do sistema $\beta$ dicetona, o oxigênio enólico em $\mathrm{C} 3$ e os nitrogênios em C4 e no grupo amida do anel A. Tetraciclinas em geral, possuem três prótons dissociáveis, um no sistema tricarbonil C1-C3 no anel A, outro no sistema ceto-fenólico em $\mathrm{C} 10-\mathrm{C} 12$, e outro no grupo dimetilamônio em $\mathrm{C} 4$, com valores de pka próximos a 3,47, 7,66 e 9,29, respectivamente [3]. Assim, controlando o $\mathrm{pH}$ do meio é possível obter diferentes 
complexos metálicos, uma vez que apenas um ou mais sítios de coordenação estarão disponíveis.

Os complexos de paládio obtidos em solução aquosa, complexos I e II, Figura 1, são sólidos bastante solúveis em DMF e DMSO. Os complexos obtidos foram caracterizados por espectroscopia na região do infravermelho e UV-Vis, $\mathrm{RMN}$ de ${ }^{1} \mathrm{H}$, por análise térmica (TG/DTG), análise elementar e condutimétrica.<smiles>C[C@@]1(O)C2=C(C(=O)[C@@]3(O)C(O)=C4C(=O)c5c(O)cccc5[C@](C)(O)[C@@H]([N+](C)(C)C)[C@H]4C(O)C13)C(N)O[Z2](Cl)(Cl)O2</smiles>

Complexo I

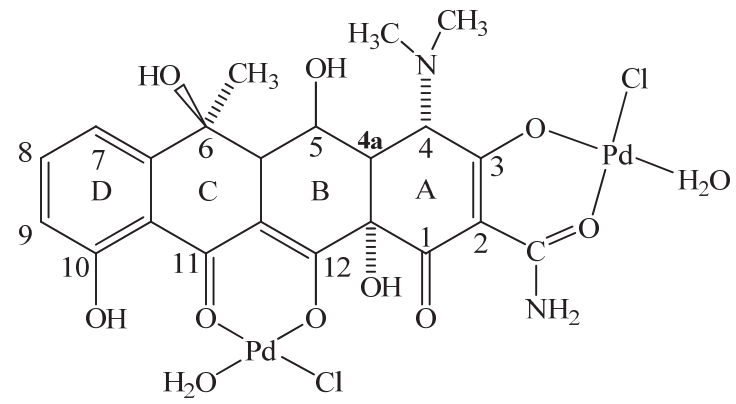

Figura 1. Estrutura proposta para os complexos obtidos.

Os resultados de análise elementar e absorção atômica corroboram com as estruturas propostas.

O Valor obtido de condutividade molar de uma solução $10^{-3} \mathrm{M}$ dos complexos em DMF a $25^{\circ} \mathrm{C}$, foram bem abaixo quando comparado ao padrão, indicando que os complexos são neutros (brometo de tetraetilamônio; $\Lambda_{\mathrm{M}}=79,56 \mu \mathrm{s} / \mathrm{cm}^{-1} /$ complexo I; $\Lambda_{\mathrm{M}}=5,56 \mu \mathrm{s} / \mathrm{cm}^{-1}$, complexo II; ; $\Lambda_{\mathrm{M}}=$ $\left.9,56 \mu \mathrm{s} / \mathrm{cm}^{-1}\right)$ [3].

A curva TG/DTG para complexo I, Figura 2, revela que o complexo perde massa entre 50-100 ${ }^{\circ} \mathrm{C}$, correspondendo $5,25 \%$ de perda de massa que corresponde à saída de duas moléculas de águas não coordenadas, (valor calculado: 5,06 \% de massa).
Entre $140-325^{\circ} \mathrm{C}$ ocorre uma série de eventos de perda de massa que se deve a decomposição térmica do complexo, saída do ligante, correspondendo a $70,14 \%$ de perda de massa (valor calculado: 69,96 $\%$ massa), no entanto, infelizmente não conseguimos atribuir estas perdas de massa, uma vez que o ligante apresenta massa molar elevada e uma estrutura bastante complexa havendo inúmeras possibilidades de quebra de ligações com formação de diversos produtos de decomposição. A partir de $600^{\circ} \mathrm{C}$ há um resíduo que corresponde a $15,40 \%$ de massa. Este resíduo foi identificado por difração de raios-X de pó como paládio elementar, número 5681 do banco de dados do ICCD (centro internacional para dados de difração), embora quantidades pequenas de óxido de paládio possam ter sido formados. A porcentagem de paládio está de acordo com a estrutura proposta (calculado: $14,97 \%$ de massa).

Para o complexo II, as curvas TG/DTG mostram um evento de perda de massa na faixa entre $45-140^{\circ} \mathrm{C}$ que corresponde à saída de duas moléculas de água coordenadas e uma não coordenada. Foram identificados outros eventos de perda de massa referentes à saída dos ligantes (decomposição do complexo), mas infelizmente não foi possível atribuir estas perdas de massa. A partir de $800^{\circ} \mathrm{C}$ observou-se a formação de um resíduo estável que foi caracterizado como Pd elementar por difração de raios-X de pó. A porcentagem de resíduo confere com a estrutura proposta para o complexo II.

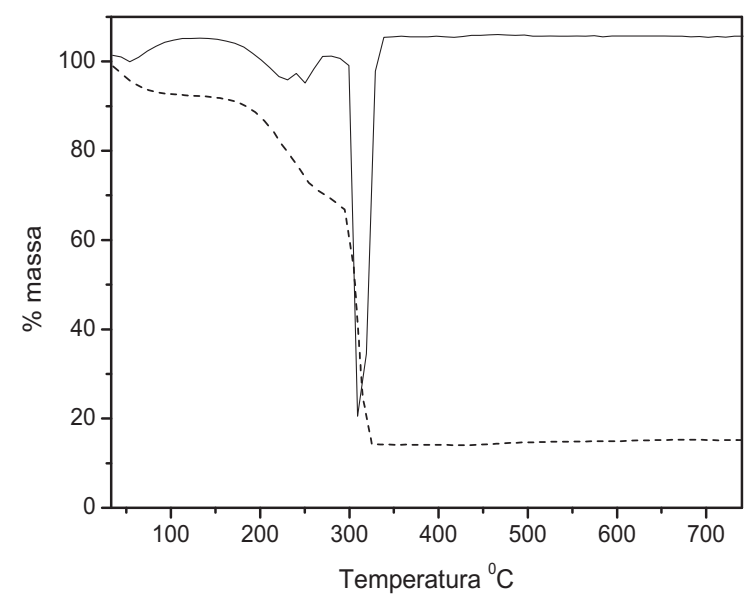

Figura 2. Curva TG/DTG para o complexo I. 
Os espectros no infravermelho dos complexos (Figura 3) foram analisados conforme o estudo detalhado feito por Dziegielewski e colaboradores para tetraciclinas [15]. A maior modificação no espectro de infravermelho do complexo I quando comparado ao ligante livre se refere à banda característica do grupo amida. A banda amida $\mathrm{I}\left(v_{\mathrm{C}=0}\right)$ que no ligante aparece em $1669 \mathrm{~cm}^{-1}$, no complexo ela se desloca para $1630 \mathrm{~cm}^{-1}$ (ombro), um deslocamento de $39 \mathrm{~cm}^{-1}$. Esta banda é muito sensível à coordenação de íons metálicos [3].

Outras duas bandas em 1616 e $1585 \mathrm{~cm}^{-1}$, também referentes ao $v_{\mathrm{C}=0}$ das carbonilas do anel Ae C, respectivamente, aparecem no complexo na mesma posição, excluindo a participação dos oxigênios destas carbonilas na coordenação do metal. Em adição, bandas na região de 3400,3100 e $3000 \mathrm{~cm}^{-1}$ são referentes aos grupos $\mathrm{OH}, \mathrm{NH}_{2} \mathrm{e} \mathrm{CH}$, respectivamente. Novas bandas de absorção observadas em 302 e 328 $\mathrm{cm}^{-1}$ são atribuídas ao $v_{\text {Pd-Cl }}$, que está de acordo com a geometria cis proposta para o complexo obtido [19]. Para o complexo II, observa-se também o deslocamento da banda referente à carbonila do grupo amida $\mathrm{e}$, além disso, a banda referente ao $v_{\mathrm{C}=0}$ do anel $\mathrm{C}$ não é observada, indicando que houve um deslocamento que é coerente com a participação deste grupo na coordenação ao metal. É interessante relembrar que esta banda não sofre alteração no espectro do complexo I. A outra banda em $1616 \mathrm{~cm}^{-1}$, referente ao $v_{\mathrm{C}=\mathrm{O}}$ da outra carbonila do anel A, apareceu no complexo na mesma freqüência, excluindo a participação deste grupo na coordenação ao metal. Uma nova absorção na região de $330 \mathrm{~cm}^{-1}$ foi atribuída ao $v_{\mathrm{Pd}-\mathrm{Cl}}$.

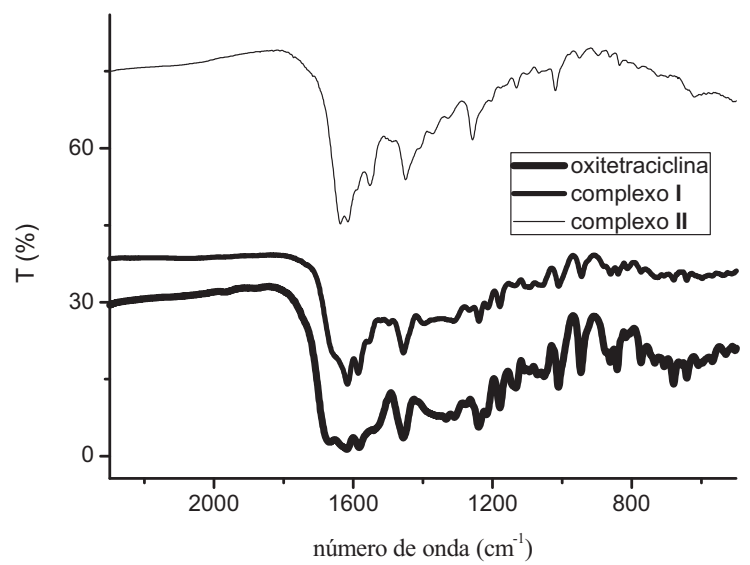

Figura 3. Seção do espectro de infravermelho da oxitetraciclina e de seus respectivos complexos.
Os espectros de absorção eletrônica para o ligante oxitetraciclina e de seus complexos de paládio(II) foram realizados. O espectro de absorção de uma solução $5 \times 10^{-5} \mathrm{M}$ de oxitetraciclina em acetonitrila revela quatro bandas de absorção centradas em 361, 314 (ombro), 265 e $218 \mathrm{~nm}$. De acordo com MCCormick et al. a transição $\pi-\pi^{*}$ do sistema tricarbonil do anel A contribui apenas com a banda em $260 \mathrm{~nm}$, enquanto a transição $\pi-\pi^{*}$ do cromóforo BCD contribui com todas, em especial a banda centrada em $361 \mathrm{~nm}$ [16]. Estas transições são sensíveis á desprotonação e complexação, ou seja, a adição de um metal coordenante modifica fortemente o espectro de absorção.

O espectro de absorção de uma solução 5 x 10${ }^{5} \mathrm{M}$ do complexo I em acetonitrila, Figura 4, revela bandas de absorção centradas em 363, 247 e 209 nm. Como podemos observar a maior modificação envolve a banda de absorção referente ao anel A. De $265 \mathrm{~nm}$ ela se desloca para $247 \mathrm{~nm}$, sugerindo o envolvimento do anel A na coordenação ao metal. A banda centrada em $363 \mathrm{~nm}$ praticamente não sofre alteração excluindo o cromóforo $\mathrm{BCD}$ de um possível envolvimento na coordenação ao metal. Já para o complexo II ocorrem modificações em todas as bandas (ver Figura 3) indicando que tanto o sistema tricarbonil do anel A e o cromóforo BCD estão envolvidos na coordenação aos íons Pd(II). No complexo bimetálico as bandas ocorrem em 233, 290 e $387 \mathrm{~nm}$.

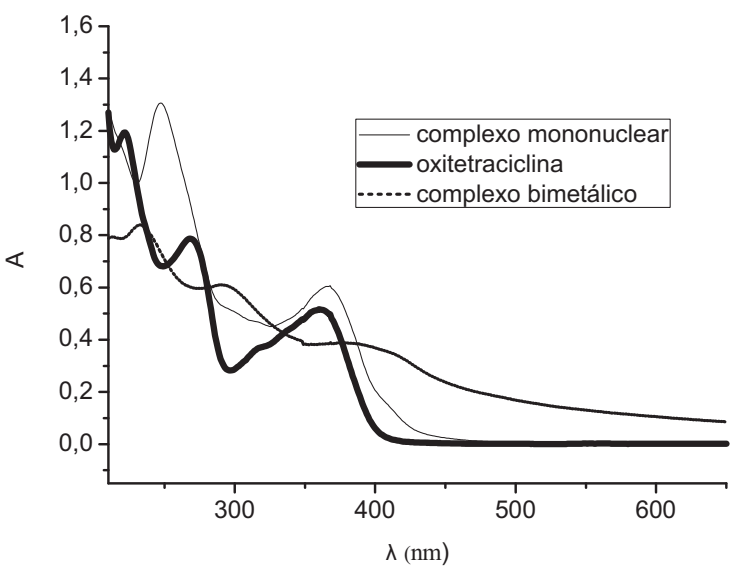

Figura 4. Espectro de absorção de uma solução 5x10-5 M do complexo I, complexo II e do ligante em acetonitrila.. 
Com os resultados obtidos, sugerimos que o oxigênio do grupo amida e o oxigênio da hidroxila ligado ao carbono 3 estão envolvidos na coordenação. Além disso, devido ao efeito quelato, a coordenação ao oxigênio ligado ao carbono 3 que encontrase desprotonado e ao oxigênio do grupo amida é mais atrativa [3]. Este modo de coordenação já foi evidenciado para o complexo de mercúrio $\left[\mathrm{Hg}\left(\mathrm{C}_{22} \mathrm{H}_{24} \mathrm{~N}_{2} \mathrm{O}_{9}\right) \mathrm{Cl}_{2}\right] \cdot 2 \mathrm{H}_{2} \mathrm{O}$ que teve sua estrutura determinada[20]. Outros trabalhos publicados na literatura também sugerem este sítio de coordenação [21]. Nosso grupo de pesquisa tem realizado estudos de RMN ${ }^{1} \mathrm{H}$ em solução aquosa contendo tetraciclinas e os íons Pd(II) e Pt(II) que também sugerem a coordenação do metal via anel $\mathrm{A}[3,22]$. Em relação ao outro sítio proposto, no caso do complexo II, o espectro no infravermelho mostra claramente o envolvimento da $\mathrm{C}=\mathrm{O}$ do anel $\mathrm{C}$. Numa tentativa de melhor elucidar a estrutura proposta para o complexo II, foi obtido seu espectro de RMN de ${ }^{1} \mathrm{H}$ utilizando DMSO como solvente. O espectro foi obtido imediatamente após a dissolução para evitar a solvólise total do complexo. Duas regiões no espectro de RMN de ${ }^{1} \mathrm{H}$ são de especial interesse para a atribuição dos sítios de coordenação, que são os prótons aromáticos do anel $\mathrm{D}$ e o sinal referente aos hidrogênios do grupo $\mathrm{N}\left(\mathrm{CH}_{3}\right)_{2}$. Estes prótons não são lábeis e seus valores de deslocamento químico $(\delta)$ são sensíveis à coordenação metálica, e assim pode-se confirmar a coordenação no anel A e no sistema ceto-fenólico, mais especificamente no oxigênio em $\mathrm{C}_{10}$ ou em $\mathrm{C}_{12}$. Os pró- tons dos grupos $\mathrm{OH}$ e do grupo amida não são observados devido à troca por deutério $[3,22]$. $\mathrm{O}$ sinal ressonância referente ao grupo $\mathrm{N}\left(\mathrm{CH}_{3}\right)_{2}$ é bastante intenso no espectro do ligante e ocorre em aproximadamente $\delta 2,85$ [22]. Caso o grupo dimetilamônio esteja desprotonado, este sinal ocorre próximo a $\delta 2,4$ uma vez que estes prótons ficam mais protegidos [23, 24]. Na Figura 5, mostramos uma seção do espectro de $\mathrm{RMN}$ de ${ }^{1} \mathrm{H}$ do complexo II, onde observamos um dupleto aproximadamente em 2,33 que é referente aos prótons metilênicos do grupo $\mathrm{N}\left(\mathrm{CH}_{3}\right)_{2}$, confirmando a desprotonação do grupo dimetilamônio. $\mathrm{O}$ fato de os sinais dos prótons do grupo dimetilamônio aparecerem desdobrados no espectro do complexo indica a presença do ligante livre e da espécie complexada [3]. No caso do complexo I, o sinal referente aos prótons metilênicos ocorrem em 2,81 indicando que o grupo $\mathrm{NH}_{3}$ está protonado. Para o complexo II, observa-se que os prótons aromáticos aparecem desdobrados, porém todos com um deslocamento químico pouco significativo, indicando a presença do ligante livre e do complexo em equilíbrio. $\mathrm{O}$ fato de haver modificações nos dois sítios de coordenação está de acordo com a proposta de coordenação de dois íons metálicos. Porém sugerimos que a coordenação ocorre no carbono C11 e no $\mathrm{C} 12$, pois se a coordenação fosse em C10 e C11 haveria um deslocamento mais significativo dos prótons aromáticos. Para o complexo I, observamos que não ocorreram mudanças nos sinais referentes aos prótons aromáticos.

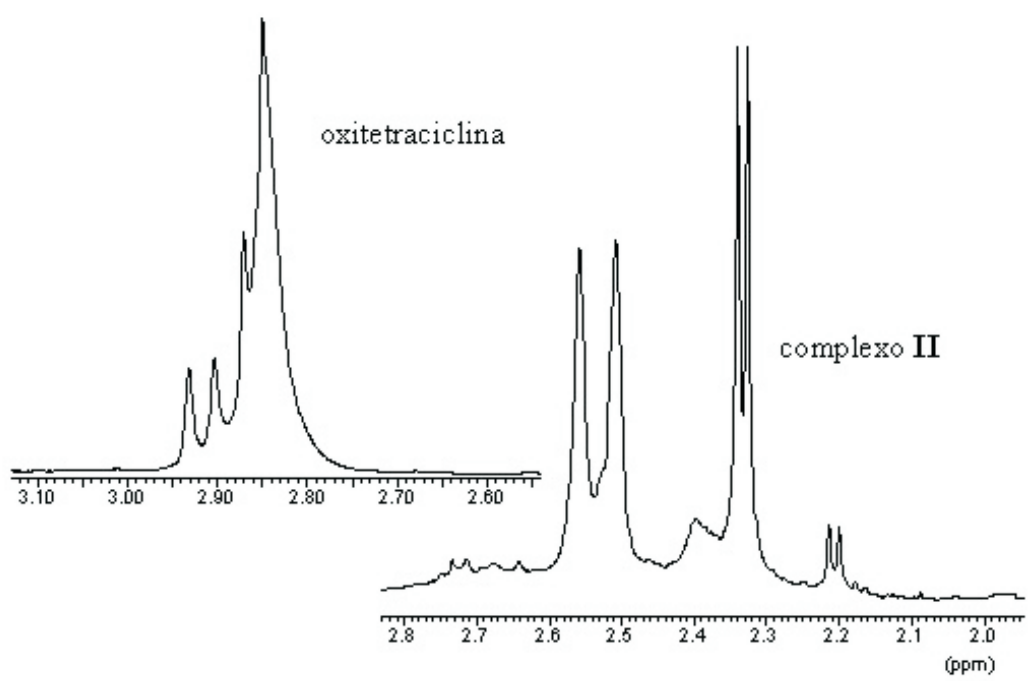

Figura 5. Seção do espectro de RMN de $1 \mathrm{H}$ do complexo II e da oxitetraciclina. 


\section{Conclusões}

Este trabalho descreve a síntese e caracterização de um novo complexo mononuclear de Pd(II) e de um novo complexo binuclear de $\operatorname{Pd}($ II) contendo oxitetraciclina como ligante. Os complexos foram caracterizados pelas técnicas usuais de análise e para o complexo bimetálico os dados espectroscópicos indicaram o envolvimento do anel A (oxigênios do grupo amida e da hidroxila em C3 após sofrer despro- tonação) e do cromóforo $\mathrm{BCD}$ (oxigênios da carbonila em C11 e da hidroxila em C12) na coordenação ao metal. Tendo em vista a estrutura dos complexos obtidos, este serão objetos de estudos posteriores.

\section{Agradecimentos}

Ao CNPq, FAPEMIG e Universidade Federal de Uberlândia pelo suporte financeiro.

Wendell Guerra, Ana Paula Soares Fontes, Elene Cristina Pereira-Maia. Synthesis and characterization of a new complex binuclear of pd (ii) containing the antibiotic oxytetracycline

Abstract: This article reports the synthesis and characterization of a new binuclear complex of palladium (II) containing the antibiotic oxytetracycline. The complex was characterized by the usual techniques of analysis. With respect to sites of coordination, the IR spectral data suggests the involvement the oxygen of the amide group and the oxygen of the neighbor hydroxyl group at ring A and to the carbonyl oxygen at $\mathrm{C} 11$ and the hydroxyl group at C12.

Keywords: palladium compounds, tetracycline, oxytetracycline.

\section{Referências}

[] I. Chopra, M. Roberts, Microbiol. Mol. Biol. Rev. 65(2) (2001) 232.

[2] B. S. Speer, N. B. Shoemaker, A. A. Salyers, Clin. Microbiol. Rev. 5(4) (1992) 387.

[3] W. Guerra, E. A. Azevedo, A. R. S. Monteiro, A. M. A. Nascimento, E.Chartone-Souza, M. BucciarelliRodriguez, A P. S. Fontes, L. Lê Moyec, E. C. PereiraMaia, J. Inorg. Biochem. 99(12) (2005) 2348.

[4] F. C. S de Paula, W. Guerra, I. R. Silva, J. N. Silveira, F. V. Botelho, L. Q. Vieira, E. C. Pereira-Maia, Chemistry \& Biodiversity 5(10) (2008), 2124.

[5] A. Garoufis, S. K. Hadjikakou, N. Hadjiliadis, Coord. Chem. Rev. 253 (2009) 1384.

[6] M. Jezowska-Bojezuk, L. Lambs, H. Kozlowski, G. Berthon, Inorg. Chem. 32 (4) (1993) 428.

[7] C. M. Mikulski, J. Fleming, D. Fleming, Inorg. Chim. Acta. 144 (1988) 9.

[8] M. Brion, L. Lambs, G. Berthon, Inorg. Chim. Acta 123 (1986) 61.

[9] G. W. Everett, Jr., D. E. Williamson, J. Am. Chem. Soc. 97 (1975) 2397.

[0] G. W. Everett, J. Gulbis, J. Am. Chem. Soc. 97 (21) (1975) 6248 .

[1] G. W. Everett, J. Gulbis, C. W. Frank, J. Am. Chem. Soc. 98 (5) (1976) 1280.

[2] L. M. Hirschy, T. F. Van Geil, J. D. Winefordner, R. N. Kelly, S. G. Schulman, Anal. Chim. Acta 166 (1984) 207219.
[3] J. A. Cowan, T. Ohyama, Inorg. Chem. 34 (11) (1995) 3083 .

[4] M. A. Ghandour, H. A. Azab, A. Hassan, Monatsh. Chem. (in germain) 123 (1992) 51.

[15] J. Dziegielewski, J. Hanuza, B. J. Trzebiatowska, Bull. Acad. Pol. Sci., Ser. Sci. Chim. 24 (1976) 307.

[16] J. R. D. McCormick, S. M. Fox, L. L. Smith, B. A. Bitler, J. Reichenthal, V. E. Origoni, W. H. Muller, R. Winterbotton, A. P. Doersclusk, J. Am. Chem. Soc. 79 (1957) 2849.

[17] A. A. M. Aly, A. Strasser, A. Vogler, Inorg. Chim. Acta 336(2002) 91

[18] A. A. M. Aly, A. Strasser, A. Vogler, Inor. Chem. Comm. 5 (6) (2002) 411.

[19] E. Chartone-Souza, T. L. Loyola, M. BucciarelliRodriguez, M. A. Menezes, N. A. Rey, E. C. Pereira-Maia, J. Inorg. Biochem. 95 (5) (2005) 1001.

[20] K. H. Jogun, J. J. Stezowski, J. Am. Chem. Soc. 98 (19) (1976) 6018 .

[21] H. F.dos Santos, B. L. Marcial, C. F De Miranda, L. A. S. Costa, W. B. De Almeida, J. Inorg. Biochem. 100 (10) (2006) 1594.

[22] W. Guerra, I. R Silva, E. A. Azevedo, A. R. S. Monteiro, M. Bucciarelli-Rodriguez, E. Chartone-Souza, J. N. Silveira, A. P. S. Fontes, E. C. Pereira-Maia, J. Braz. Chem. Soc. 17 (8) (2006) 1627.

[23] A. F Casy, A. Yasin, J. Pharm. Biomed. Anal. 1 (1983) 281.

[24] A. F. Casy, A. Yasin, Magn. Res. Chem. 23 (9) (1985)

767. 